\title{
Family and peer effect on young and adolescent smoking in Bangladesh
}

\section{CURRENT STATUS: POSTED}

Research Square

Moin Uddin Ahmed

Western Sydney University

moin.ahmed@westernsydney.edu.auCorresponding Author

ORCiD: https://orcid.org/0000-0002-1733-5216

Rubayyat Hashmi

University of Southern Queensland Faculty of Business Education Law and Arts

Mohammad Habibullah Pulok

Nova Scotia Health Authority

Kathy Tannous

Western Sydney University

Khorshed Alam

University of Southern Queensland Faculty of Business Education Law and Arts

\section{DOI:}

$10.21203 /$ rs.2.20836/v2

\section{SUBJECT AREAS}

Health Economics \& Outcomes Research Health Policy

\section{KEYWORDS}

tobacco, smoking, adolescent, young adults, peer, family, Bangladesh 


\section{Abstract}

The authors have withdrawn this preprint from Research Square. 